\title{
On the Apparent SEC Molecular Weight and Polydispersity Reduction upon Intramolecular Collapse of Polydisperse Chains to Unimolecular Nanoparticles
}

\author{
José A. Pomposo ${ }^{1,2,3}$ *, Irma Perez-Baena ${ }^{1}$, Lorea Buruaga ${ }^{1}$, Angel Alegría ${ }^{1,3}$, Angel J. \\ Moreno ${ }^{l}$ and Juan Colmenero ${ }^{1,3,4}$
}

${ }^{1}$ Centro de Física de Materiales (CSIC, UPV/EHU) - Materials Physics Center, Paseo Manuel de Lardizabal 5, 20018 San Sebastián, Spain. ${ }^{2}$ IKERBASQUE - Basque Foundation for Science, Alameda Urquijo 36, 48011 Bilbao, Spain. ${ }^{3}$ Departamento de Física de Materiales, Universidad del País Vasco (UPV/EHU), Apartado 1072, 20800 San Sebastián, Spain. ${ }^{4}$ Donostia International Physics Center, Paseo Manuel de Lardizabal 4, 20018 San Sebastián, Spain

E-mail: josetxo pomposo@ehu.es

ABSTRACT: When unimolecular particles of molecular weight $M$ are formed by intramolecular cross-linking of individual polymer chains, a significant reduction in hydrodynamic radius and, hence, apparent molecular weight $\left(M_{a p p}\right)$ is found by size exclusion chromatography (SEC) with traditional calibration. A concomitant reduction in polydispersity index $(P D I)$ is often observed. This work tries to clarify the relationship between the molecular weight decrease and the simultaneous $P D I$ narrowing reported by SEC for collapsed polymeric nanoparticles. A powerlaw expression is derived by assuming that fractal, soft nano-objects are formed upon intramolecular collapse: $M_{a p p} \propto M^{\beta}$ where $\beta=\left(1+\alpha_{F}\right) /\left(1+\alpha_{L}\right), \alpha_{F}$ being a parameter related to the fractal degree of the soft nanoparticle and $\alpha_{L}$ the Mark-Houwink-Sakurada exponent of the precursor polymer, respectively. Two limiting cases provide the expected minimum and maximum $\beta$ values: $\beta \approx 0.56$ for perfectly compact nanoparticles and $\beta \approx 1$ for nano-objects equivalent to flexible chains in good solvent, respectively. Results available in the literature for intramolecular cross-linked nanoparticles of different chemical composition have been analyzed in terms of this simple scaling law. Its direct effect on SEC polydispersity reduction is addressed for a precursor displaying a log-normal molecular weight distribution. The apparent polydispersity of the nanoparticles is given by $P D I_{a p p}=(P D I)^{\beta^{2}}$. Due to the heterogeneous nature of the collapsing process and/or the potential presence of marginal, minor amounts of intermolecular byproducts, some deviation of experimental $P D I_{a p p}$ data from theoretical predictions is observed. 


\section{Introduction}

The design of functional soft nanoparticles is of current interest for several emerging fields due to the exceptional and sometimes unique properties displayed by such nanoobjects. ${ }^{1}$ Among others, the irreversible intramolecular collapse of individual polymer chains to unimolecular nanoparticles in the sub-20 nm size range has become an efficient route for the synthesis of functional soft nano-objects. ${ }^{2}$ Interestingly, the intramolecular collapsing process of synthetic polymers resembles to some extent the cooperative folding process found in natural proteins. Potential applications for singlechain nanoparticles cover from processing additives ${ }^{3}$ to artificial enzymes ${ }^{4}$, photostable bio-imaging agents ${ }^{5}$ and drug/siRNA-delivery systems ${ }^{6}$, among others.

In general, the evolution of the size reduction at different stages of intramolecular cross-linking of individual chains to single-chain nanoparticles has been followed mainly by size exclusion chromatography (SEC), ${ }^{1 \mathrm{~d}, 2,7-20}$ dynamic light scattering ${ }^{1 \mathrm{~d}, 2,7,9,13,15-19,21-23}$ and viscosimetry. ${ }^{15,17,22}$ The morphology of the resulting nanoparticles due to the chain collapse process has been investigated by field emission scanning electron microscopy (FE-SEM), ${ }^{7,8}$ atomic force microscopy (AFM) ${ }^{1 \mathrm{~d}, 9-11,16,18-}$ 20,22 and transmission electron microscopy $(\mathrm{TEM})^{4 \mathrm{a}, 12,13,15,16,21,23}$ whereas the crosslinking degree has been determined qualitatively by Fourier transform infra-red spectroscopy $^{1 \mathrm{~d}, 13,15,17}$ and quantitatively by nuclear magnetic resonance spectroscopy. ${ }^{1 \mathrm{~d}, 2,7-23}$ Thermal properties of the resulting nanoparticles have been determined mostly by differential scanning calorimetry ${ }^{7,11,20,23}$ and thermogravimetric analysis. ${ }^{1 d}$ Additionally, in some special cases, the intramolecular reaction process has been monitored by ultraviolet-visible ${ }^{10,19,21}$ and photoluminescence ${ }^{1 \mathrm{~d}, 18}$ spectroscopies. Nanoparticle absolute molar mass has been traditionally monitored by static light scattering following conventional Zimm procedures. ${ }^{2,16,24}$

From an experimental point of view, it is a highly-demanding task to prepare single-chain particles by internal crosslinking without any intermolecular crosslinking taking place, although several strategies have been implemented to severely minimize nanoparticle cross-coupling. Currently, intramolecular cross-linked polymeric nanoparticles are mainly synthesized following the so-called continuous addition technique $\mathrm{e}^{1 \mathrm{~d}, 2,5 \mathrm{c}, 12-15,17,18,23}$ since it minimizes inter-chain reactions (i.e. absolute molar mass of the resulting nanoparticle very similar to that of the linear precursor) allowing 
single-chain nanoparticles to be produced in large quantities. This has been confirmed experimentally by Hawker and coworkers ${ }^{2}$ which found that the actual molecular weight of polystyrene nanoparticles as determined by static light scattering (LS) was very similar to that of the starting linear precursors. Similar agreement between absolute molar mass by LS, before and after reaction, has been reported for other collapsed unimolecular nano-objects of more complex architecture (e.g. $\operatorname{tadpole}^{24}$ and Janus ${ }^{16}$ nanoparticles).

SEC has become a very powerful technique to distinguish between intramolecular and intermolecular events during the synthesis of unimolecular nanoparticles. ${ }^{25}$ Traditionally, SEC has been a technique very valuable to discriminate linear macromolecules according to their hydrodynamic radius $\left(R_{H}\right)$ and hence to quantify the $R_{H}$ size distribution of a given sample. ${ }^{26}$ As expected, a significant reduction in $R_{H}$ (i.e. a significant increase in retention time, $t_{R}$ ) is found by SEC with traditional calibration upon chain collapse..$^{2,7-20}$ A concomitant reduction in polydispersity is often observed even when the actual molecular weight distribution should remain largely unaltered or even broadened if intermolecular cross-linking takes place.

This work tries to clarify the relationship between the "apparent" SEC molecular weight decrease and the simultaneous polydispersity narrowing observed upon collapse of polydisperse chains to unimolecular nanoparticles. Hence, a power-law expression for the apparent molecular weight reduction depending on the starting precursor molecular weight and the resulting nanoparticle structure is derived by assuming that fractal, soft nano-objects are formed upon intramolecular collapse. Two limiting cases are considered for arriving at the maximum and minimum values of the power-law exponent: on the one hand, perfectly compact (non-fractal) spheres and, on the other hand, nano-objects showing a fractal behavior identical to swollen linear polymer chains in a good solvent. Results available in the literature for collapsed nanoparticles of different chemistries (styrene-, methacrylate-, caprolactone-, $n$-butyl acrylate-, ethylene glycol/styrene-, carbonate- and benzyl acrylate-based nanoparticles) are then analyzed in terms of this simple scaling law for fractal, soft nanoparticles. Finally, its direct effect on polydispersity reduction is clearly illustrated by comparing the experimental and theoretical apparent SEC polydispersity values for different intramolecular cross-linked nanoparticle systems reported in the literature. 


\section{Apparent molecular weight upon unimolecular nanoparticle formation}

We are interested in deriving an expression for the expected "apparent" SEC molecular weight $\left(M_{\text {app }}\right)$ of unimolecular nanoparticles synthesized form linear polymeric precursors by intramolecular cross-linking reactions. Let us assume that upon irreversible collapse of a polymeric precursor of molecular weight $M$ and density $\rho$ a fractal, soft nanoparticle is obtained. The hydrodynamic radius of such a nano-object, $R_{H}$, can be expressed under good solvent conditions as: ${ }^{27}$

$$
R_{H}=q_{F} M^{\left(1+\alpha_{F}\right) / 3}
$$

where $q_{F}$ is a constant, and $\alpha_{F} \geq 0$ is a parameter related to the fractal nature of the nanoparticle which measures its departure from the ideal "hard-sphere" state. Two limiting cases of eq. 1 are worth of mention: i) perfectly compact spheres (CS), for which $\alpha_{F}=\alpha_{F}^{C S}=0$ and $q_{F}=q_{F}^{C S}=\left(\frac{3}{4 \pi N_{A} \rho}\right)^{1 / 3}$, where $N_{A}$ is Avogadro's number and ii) nano-objects showing a fractal behavior very similar to that of linear polymer (LP) chains in good solvents, for which $\alpha_{F}=\alpha_{F}^{L P} \approx \alpha_{L}$ and $q_{F}=q_{F}^{L P} \approx q_{L}=\left(\frac{3 K}{10 \pi N_{A}}\right)^{1 / 3}$, where $\alpha_{L}$ and $K$ are Mark-Houwink-Sakurada constants. In general, for unimolecular fractal nanoparticles one expects: $0 \leq \alpha_{F} \leq \alpha_{L} \approx 0.8$ (i.e. flexible chains, good solvent).

During SEC analysis with traditional calibration, the hydrodynamic radius of the collapsed nanoparticle will be assimilated to that of an equivalent flexible chain of identical hydrodynamic radius, intrinsic viscosity $[\eta]=K M_{a p p}^{\alpha_{L}}$ and molecular weight $M_{\text {app }}$, according to:

$$
R_{H}=q_{L} M_{a p p}^{\left(1+\alpha_{L}\right) / 3}
$$

Conceptually, the nanoparticle is hence "replaced" by an equivalent (nonfunctionalized) linear polymer chain of identical hydrodynamic radius and molecular weight $M_{a p p}$. By combining equations 1 and 2 we obtain:

$$
M_{a p p}=c M^{\beta}
$$


where $c$ is a constant given by $c=\left(q_{F} / q_{L}\right)^{3 /\left(1+\alpha_{L}\right)}$ and the power-law exponent is:

$$
\beta=\left(\frac{1+\alpha_{F}}{1+\alpha_{L}}\right)
$$

According to the above analysis, we have estimated a lower value of $\beta \approx 0.56$ for compact, uniform particles $\left(\alpha_{F}=0, \alpha_{L} \approx 0.8\right)$ and an upper value of $\beta \approx 1$ for nanoobjects showing a fractal behavior very similar to swollen flexible chains. In general, one therefore expects: $0.56 \leq \beta \leq 1$.

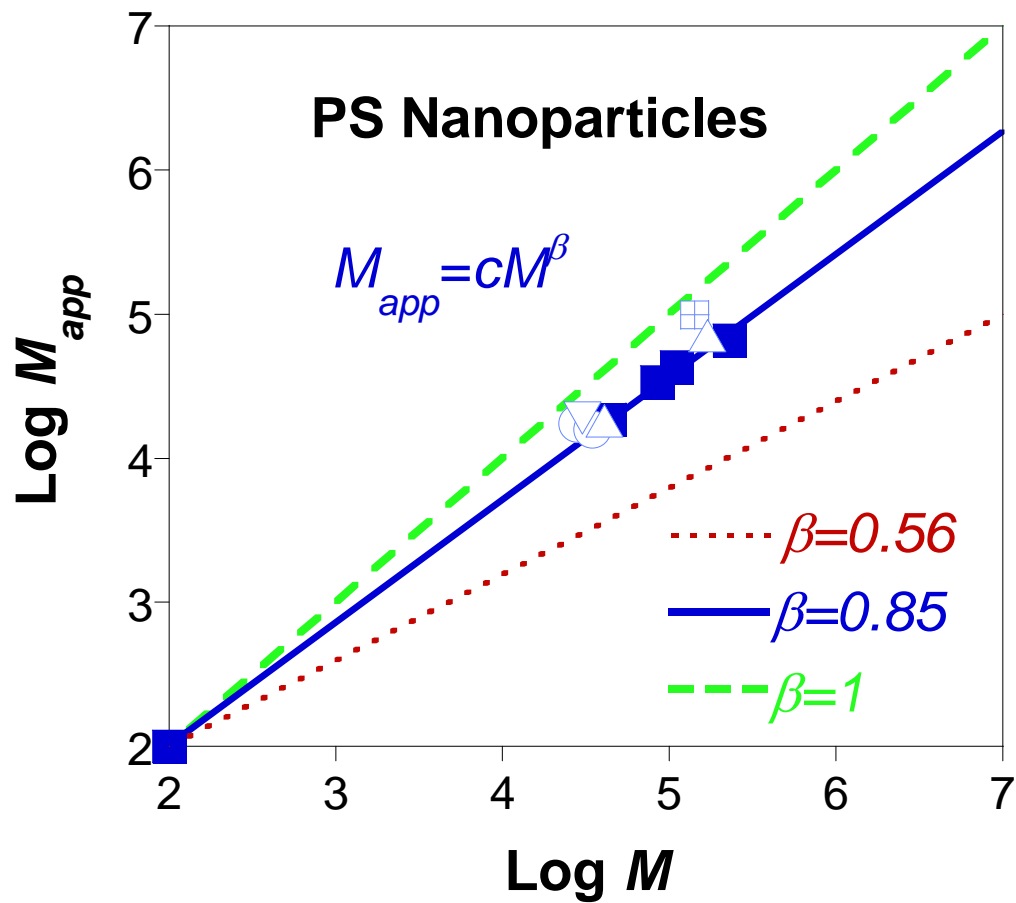

Figure 1. Apparent molecular weight $\left(M_{a p p}\right)$ of PS nanoparticles versus molecular weight $(M)$ of the corresponding PS precursor. Squares, triangles, circles, inverted triangles and crossed squares are experimental data from references 2, 7, 9, 12 and 18, respectively. Dotted, continuous and dashed lines correspond to uniform, compact spheres $(\beta=0.56)$, fractal nanoparticles $(\beta=0.85)$ and nano-objects similar to swollen polymer chains $(\beta=1)$, respectively (see text for details).

Figure 1 illustrates experimental $M_{a p p}$ versus $M$ data from the literature for intramolecular, irreversibly cross-linked polystyrene (PS)-nanoparticles. Data in this figure cover PS-nanoparticles synthesized by different chemical approaches including 
intramolecular cross-linking of vinyl, ${ }^{7,9}$ benzocyclobutene ${ }^{2}$, o-quinodimethane ${ }^{12}$ and benzosulfone ${ }^{18}$ functional groups. Solid symbols in Fig. 1 correspond to data in which the absolute molar mass by LS of the nanoparticles was reported and resulted to be in agreement, within experimental error, with the molar mass by SEC of the linear precursor. $^{2}$ These results cover a rather broad range in $M$ corresponding to PSnanoparticles synthesized from precursor polymers containing $20 \mathrm{~mol} \%$ of cross-linking units and polydispersity $(P D I)$ values less than 1.25. Data for PS-nanoparticles for which absolute molar mass by LS was not reported ${ }^{7,9,12}$ are distinguished by open symbols. Data from ref. 18 referring to relatively high polydisperse PS nanoparticles $\left(P D I_{a p p}=2\right)$ containing an internal short fluorene rigid-rod core are distinguish by crossed squares in Fig. 1. In general, data for PS-nanoparticles for which no reliable absolute molar mass is available (open symbols) follow the same trend that the data corresponding to single-chain, unimolecular PS-nanoparticles (solid symbols).

In order to fit the data of Fig. 1 (solid symbols) by eq. 3 we have considered that $M_{\text {app }}=M$ at the molecular weight of a monomer, $M_{0} \approx 10^{2} \mathrm{Da}$. This seems to be a reasonable assumption because polymer-like behavior cannot be expected at the monomer scale. With this constraint, a value of $\beta=0.85$ is obtained from Fig. 1 by data-fitting. Anyway, by changing this criterion to $M_{a p p}=M$ at $M \approx 500 \mathrm{Da}$ corresponding to a hypothetical precursor polymer with $20 \mathrm{~mol} \%$ of cross-linking functional monomers and hence a single cross-linking monomer per chain (i.e. no selfcross-linked nanoparticles could be obtained from this precursor polymer) only a minor change in the $\beta$ value is observed ( $\beta=0.83$ ). Consequently, we can retain our prior assumption of $M_{\text {app }}=M$ at $M \approx 10^{2}$ Da resulting in $\beta=0.85$ with an estimated uncertainty of about $2 \%$. This value of the power-law exponent is far from the value of perfectly compact, non-fractal nanoparticles ( $\beta=0.56$, dotted line in Figure 1$)$ but also different from that expected for nano-objects with a fractal behavior similar to that of swollen polymer chains ( $\beta=1$, dashed line in Figure 1). From eq. 4, a value of the fractal parameter $\alpha_{F}=0.47$ is obtained by using $\beta=0.85$ and $\alpha_{L}=0.734$ for PS chains in tetrahydrofuran (THF). ${ }^{28}$ Such a $\alpha_{F}$ value is close to that commonly found for linear polymer chains in $\theta$-solvents $\left(\alpha_{L}^{\theta} \approx 0.5\right) .{ }^{29}$ This suggests that intramolecular crosslinking in good solvent effectively screens the excluded volume interactions of the 
swollen precursor, leading to nearly gaussian conformations for the resulting nanoparticles. $^{29}$

It is also interesting to compare experimental results for cyclic PS-chains ${ }^{30}$ with the predictions about "equivalent" PS-nanoparticles having a single intramolecular cross-linking point. By assuming $20 \mathrm{~mol} \%$ of cross-linking functional monomers, PSnanoparticles with a single cross-linking point should be potentially obtained from oligomeric PS chains having 10 repeat units $\left(M \approx 10^{3} \mathrm{Da}\right)$. Based on eq. $3(\beta=0.85)$ we obtain $M_{a p p} \approx 720$ Da for hypothetical PS-nanoparticles with a single cross-linking point arising from PS precursor chains of $M=10^{3} \mathrm{Da}$. On the other hand, the ratio of intrinsic viscosities of cyclic and linear PS chains was reported to be 0.71 in good solvent $^{30}$ which translates to $M_{a p p} \approx\left(0.71^{1 / 0.734}\right) M=630$ Da for cyclic PS chains synthesized from linear PS chains of $M \approx 10^{3} \mathrm{Da}$. The main difference between both systems arises as a consequence of the random placement of the two cross-linking units in the case of PS nanoparticles when compared to the bonding between chain-ends in the case of cyclic PS chains. Giving the approximations involved, there is a good agreement between predictions for collapsed PS nanoparticles having a single intramolecular cross-linking point and experimental data for cyclic PS chains.

Figure 2 shows experimental $M_{\text {app }}$ versus $M$ data for intramolecular cross-linked poly(methyl methacrylate) (PMMA)-nanoparticles. We have included data for PMMAnanoparticles obtained from different irreversible cross-linking routes covering from free-radical cross-linking of vinyl units, ${ }^{7,8}$ thermal cross-linking of benzocyclobutene groups ${ }^{2}$ and alkyne-azide "click" chemistry. ${ }^{13}$ Unfortunately, data about absolute molar mass by LS for most of these PMMA-nanoparticles are not available in the literature. Results for nanoparticles synthesized by means of supramolecular (reversible) interactions ${ }^{17}$ and for nanoparticles showing strong hydrogen bonding interactions such as those prepared by intramolecular cross-linking of isocyanate functionalized copolymers with diamines ${ }^{20}$ have not been included in Fig. 2 because eq. 3 has been derived based on the assumption of irreversible intramolecular cross-linking formation and absence of inter-particle directional interactions. The effect of supramolecular cross-links and strong specific interactions on nanoparticle apparent molecular weight and polydispersity is certainly outside of the scope of the present paper as it will require a different theoretical approach involving dynamic equilibria. 


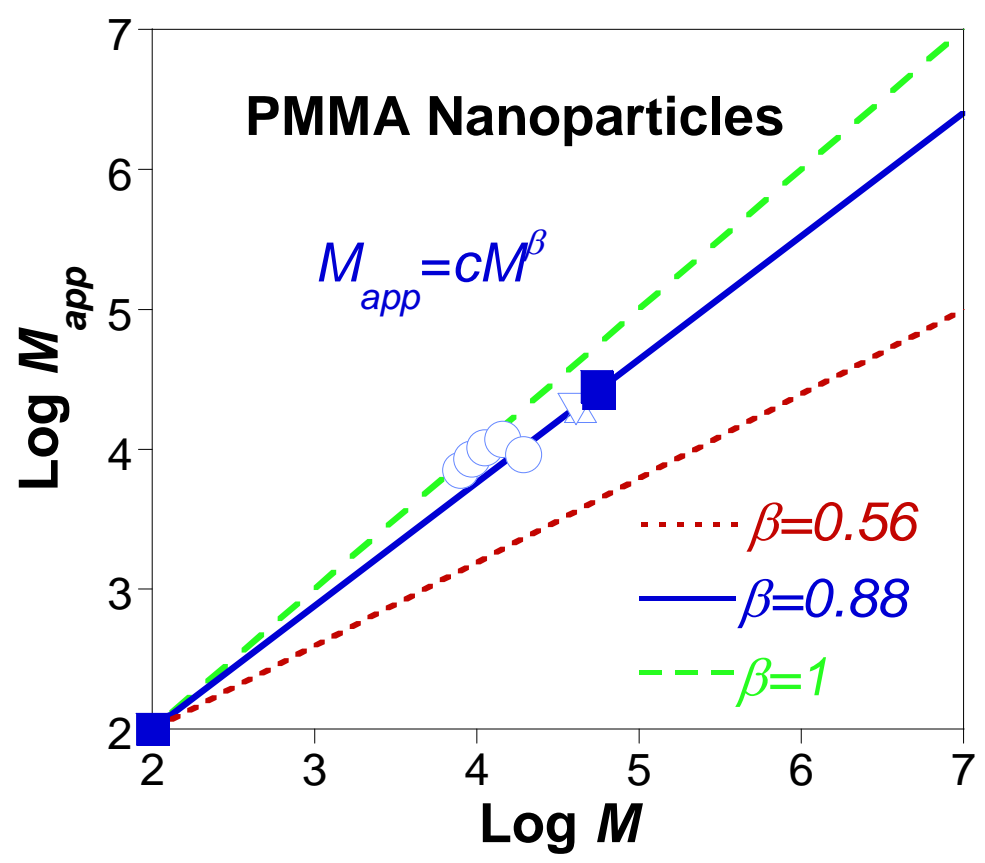

Figure 2. Apparent molecular weight $\left(M_{\text {app }}\right)$ of PMMA nanoparticles versus molecular weight $(M)$ of the corresponding PMMA precursor. Squares, triangles, circles and inverted triangles are experimental data from references $2,7,8$ and 13 , respectively. Dotted, continuous and dashed lines correspond to uniform, compact spheres $(\beta=0.56)$, fractal nanoparticles $(\beta=0.88)$ and nano-objects similar to swollen polymer chains $(\beta=$ 1), respectively (see text for details).

A data-fitting procedure similar to that performed in Fig. 1 gives $\beta=0.88$ which is very close to the value obtained for PS-nanoparticles, although a larger data scatter is visible in Fig. 2. For PMMA-nanoparticles, the corresponding fractal parameter is estimated to be $\alpha_{F}=0.52$ by using $\alpha_{L}=0.731$ for PMMA in THF. ${ }^{31}$ Interestingly, the value of the fractal parameter for PMMA-nanoparticles is also found to be similar to that of linear chains under $\theta$-solvent conditions. ${ }^{29}$ Since accurate data corresponding to the apparent SEC molecular weight of cyclic PMMA chains have been reported very recently, ${ }^{32}$ it seems appropriate to compare the results for cyclic PMMA-chains with the predictions for hypothetical PMMA-nanoparticles with a single cross-linking point. On the one hand, from eq. $3(\beta=0.88)$ a value of $M_{a p p}=761 \mathrm{Da}$ is estimated for PMMAnanoparticles with a single cross-linking point arising potentially from linear PMMA chains of $M=10^{3} \mathrm{Da}$. On the other hand, Glassner et $a l .{ }^{32}$ have reported a ratio of the apparent molecular weight of cyclic PMMA chains to the molecular weight of the linear 
PMMA precursor chains of $M_{a p p} / M=0.8$, providing $M_{a p p}=800$ Da for cyclic PMMA chains obtained from linear PMMA chains of $M \approx 10^{3} \mathrm{Da}$. Once again, a good agreement is observed between the predictions for collapsed nanoparticles having a single intramolecular cross-linking point and experimental data for cyclic chains, in spite of the presumably different topological structures involved.

Table 1 summarizes experimental $M_{\text {app }}$ versus $M$ data for other irreversibly collapsed nanoparticles of different chemical nature reported in the literature, including

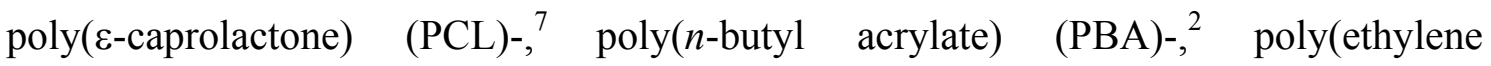
glycol/styrene) (PEGS)-, ${ }^{2}$ poly(carbonate) (PC)-, ${ }^{11}$ and poly(benzyl acrylate) (PBzA)based nanoparticles $^{12}$. We have included for comparison $M_{a p p}$ data calculated from eq. 3 by assuming, to a first approximation, that $\alpha_{F} \approx 0.5, \alpha_{L} \approx 0.7$ and $M_{a p p}=M$ at a molecular weight of $M_{0} \approx 10^{2}$ Da for all the above systems. Given the assumptions involved, the agreement between experimental and calculated data is reasonably good, especially for polymer nanoparticles synthesized from linear precursor polymers with high content of cross-linking units (>15 mol\%).

Table 1. Comparison of experimental and calculated apparent SEC molecular weight, $M_{a p p}$, for several intramolecular cross-linked nanoparticles reported in the literature.

\begin{tabular}{|c|c|c|c|c|}
\hline System & $\begin{array}{c}\text { Cross-linking } \\
\text { (mol\%) }^{\mathbf{a}}\end{array}$ & $\begin{array}{c}\boldsymbol{M} \text { (kDa) } \\
\mathbf{E x p .}^{\mathbf{b}}\end{array}$ & $\begin{array}{c}\boldsymbol{M}_{\text {app }} \text { (kDa) } \\
\mathbf{E x p}^{\mathbf{c}}\end{array}$ & $\begin{array}{c}\boldsymbol{M}_{\text {app }} \text { (kDa) } \\
\text { Calc. }^{\mathbf{d}}\end{array}$ \\
\hline $\mathrm{PCL}^{7}$ & 15 & 20.5 & 15.7 & 10.8 \\
\hline $\mathrm{PBA}^{2}$ & 20 & 73.0 & 27.8 & 32.9 \\
\hline $\mathrm{PEGS}^{2}$ & 20 & 89.5 & 36.5 & 39.4 \\
\hline $\mathrm{PC}^{11}$ & 38 & 64.9 & 37.5 & 29.7 \\
\hline $\mathrm{PBzA}^{12}$ & 5 & 68.0 & 42.0 & 30.9 \\
\hline
\end{tabular}


${ }^{a}$ Content of cross-linking units in the linear precursor polymer. ${ }^{b}$ Weight-average molecular weight of the linear precursor as determined by SEC with traditional calibration. ${ }^{\mathrm{c}}$ Weightaverage molecular weight of the resulting intramolecular cross-linked nanoparticles as determined by SEC with traditional calibration. ${ }^{\mathrm{d}}$ Calculated from equation 3 by assuming $\alpha_{F} \approx 0.5, \alpha_{L} \approx 0.7$ and $M_{a p p}=M$ at a molecular weight of $M_{0} \approx 10^{2}$ Da.

\section{Polydispersity narrowing upon unimolecular nanoparticle formation}

As a direct consequence of the validity of equation 3 for quantifying the apparent molecular weight decrease upon intramolecular chain collapse, a reduction in the apparent polydispersity index $(P D I)$ is expected, as we will show below. For the sake of simplicity, let us assume that the molecular weight distribution $(M W D)$ function of the linear polymeric precursor follows a log-normal function such as: ${ }^{33}$

$$
W(M)=\frac{1}{M \sigma_{1} \sqrt{2 \pi}} \exp \left[-\frac{\left(\ln M-\mu_{1}\right)^{2}}{2 \sigma_{1}^{2}}\right]
$$

where $\sigma_{1}$ and $\mu_{1}$ are the parameters that control the $M W D$ according to:

$$
\begin{aligned}
& \bar{M}_{n}=\exp \left[\mu_{1}+\frac{1}{2} \sigma_{1}^{2}\right] \\
& \bar{M}_{w}=\exp \left[\mu_{1}+\frac{3}{2} \sigma_{1}^{2}\right] \\
& P D I \equiv \frac{\bar{M}_{w}}{\bar{M}_{n}}=\exp \left[\sigma_{1}^{2}\right]
\end{aligned}
$$

Upon intramolecular collapse of the linear precursor, a shift in the $M W D$ is expected from $W(M)$ to $W\left(M_{a p p}\right)$ according to the above $M_{a p p}=c M^{\beta}$ scaling law (eq. 3). Hence, eq. 5 becomes:

$$
W\left(M_{a p p}\right)=\frac{1}{M_{a p p} \sigma_{2} \sqrt{2 \pi}} \exp \left[-\frac{\left(\ln M_{a p p}-\mu_{2}\right)^{2}}{2 \sigma_{2}^{2}}\right]
$$

where $\sigma_{2}=\beta \sigma_{1}$ and $\mu_{2}=\ln c+\beta \mu_{1}$.

\section{Consequently:}




$$
P D I_{a p p}=(P D I)^{\beta^{2}}
$$

Since $\beta<1$, the apparent SEC polydispersity is expected to decrease upon unimolecular nanoparticle formation. Note that according to eq. 10 the apparent polydispersity index of the nanoparticles depends only on the scaling-law exponent $\beta$ and not on the prefactor $c$.

Figure 3 provides a comparison of theory and experimental data from the literature for intramolecular cross-linked PS and PMMA nanoparticles based on eq. 10 and the respective values of the $\beta$ parameter determined previously (see Figs. 1 and 2). At this point it is worth commenting two contributions to polydispersity which are not explicitly accounted for in the former scheme. These originate from the inherent heterogeneous nature of the intramolecular cross-linking process and the potential presence of residual, minor amounts of intermolecular byproducts in some systems.

Hence, intramolecular cross-linking is a statistical process taking place inside each polymer chain in which formation of a given bond has a strong influence on the reactivity of its neighbors. Due to conformational fluctuations during chain collapse at constant temperature, certain differences in reactivity along the chain are expected even for chains having exactly the same length. Furthermore, the cross-linking process introduces severe topological constraints in orientation and distance between crosslinking groups along the chain that reduce subsequent intramolecular reactivity. This inhibition of the cross-linking process is more apparent at the late stages of the intramolecular chain collapse where often due to rigidity effects there is a fraction of cross-linking groups that are never able to find a partner. ${ }^{34}$ Consequently, topological "freezing" is expected to contribute to a relative increase in PDI when compared to theoretical predictions. The reason for this increase is that different nanoparticles will show different fractions of unreacted cross-linkers. Having noted this, it must be stressed that even for the ideal case in which all cross-linking groups have reacted and formed a permanent bond, the resulting nanoparticles will exhibit different topologies as a consequence of the stochastic character of the cross-linking process. Unlike for the precursor polymer this feature will lead, for a fixed molecular weight, to an intrinsic polydispersity in the size (and hence in the apparent mass) of the unimolecular nanoparticles. Preliminar results from molecular dynamics simulations, to be presented elsewhere, seem to confirm this feature. 

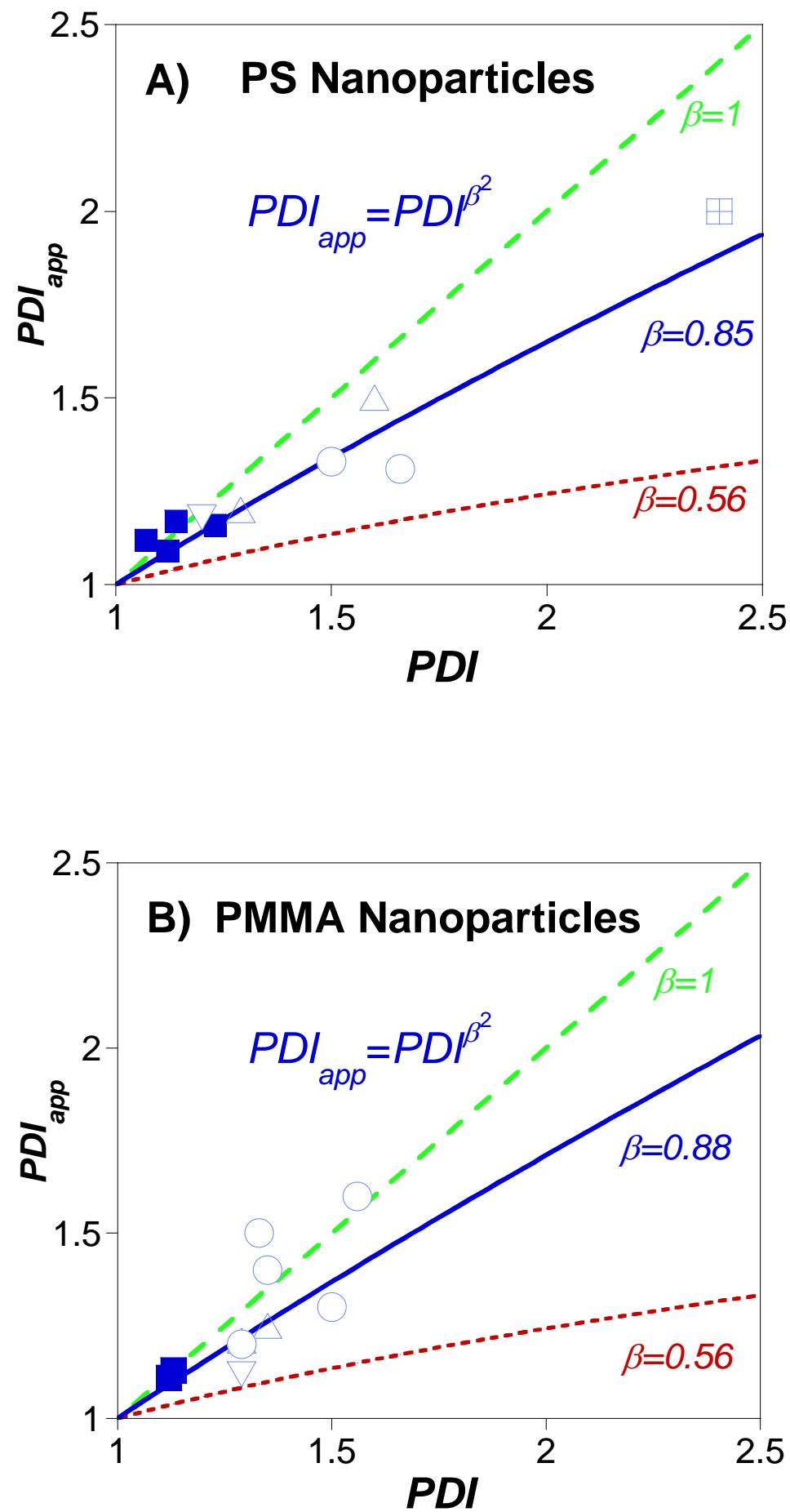

Figure 3. Apparent polydispersity index $\left(P D I_{a p p}\right)$ of intramolecular cross-linked nanoparticles versus polydispersity index $(P D I)$ of the corresponding polymeric precursors for: A) PS nanoparticles and B) PMMA nanoparticles. Identical symbols to those used in Figs. 1 and 2 are employed. Predictions from $P D I_{a p p}=P D I^{\beta^{2}}$ at different values of $\beta$ are also drawn as dashed, continuous and dotted lines (see text for details). 
Moreover, $P D I$ is a parameter very sensitive to the presence of high molecular weight tails arising from minor quantities of byproducts generated by intermolecular secondary reactions. ${ }^{2}$ In particular the strong scattering of the experimental data observed in Fig. 3B could be also tentatively attributed to the presence of residual, minor amounts of intermolecular byproducts, especially for nanoparticles synthesized by techniques differing from the so-called continuous addition technique.

Table 2 provides a comparison of $P D I_{a p p}$ versus $P D I$ data for other irreversibly collapsed nanoparticles of different chemical nature (PCL-,${ }^{7}$ PBA-,${ }^{2}$ PEGS-,${ }^{2}$ PC-,${ }^{11}$ and PBzA-based nanoparticles ${ }^{12}$ ) as well as theoretical predictions from eq. 10 by using, to a first approximation, $\beta=0.88$ (i.e., by using $\alpha_{F}=0.5$ ). In general, eq. 10 is able to reproduce the experimental trend rather well in spite of the several approximations involved, though systematic deviations are found, with the experimental values of $P D I_{a p p}$ being somewhat higher than the theoretical values. Thus, the aforementioned effects related to heterogeneity and/or intermolecular reactions might partially compensate the reduction of $P D I$ predicted by eq. 10. Accurate experimental data-sets corresponding to well-defined precursor polymers and unimolecular nanoparticles of other chemical structures, varying cross-linking degree, SEC molecular weight and polydispersity will be certainly very valuable to establish the validity range of both eq. 3 and eq. 10.

Table 2. Comparison of experimental and calculated apparent SEC polydispersity, $P D I_{a p p}$, for several intramolecular cross-linked nanoparticles reported in the literature.

\begin{tabular}{|c|c|c|c|c|}
\hline System & $\begin{array}{c}\text { Cross-linking } \\
\text { (mol\%) }^{\mathbf{a}}\end{array}$ & $\begin{array}{c}\text { PDI } \\
\text { Exp. }^{\mathbf{b}}\end{array}$ & $\begin{array}{c}\boldsymbol{P D I}_{\text {app }} \\
\text { Exp. }^{\mathbf{c}}\end{array}$ & $\begin{array}{c}\boldsymbol{P D I}_{\text {app }} \\
\text { Calc. }^{\mathbf{d}}\end{array}$ \\
\hline PCL $^{7}$ & 15 & 1.35 & 1.35 & 1.26 \\
\hline PBA $^{2}$ & 20 & 1.09 & 1.10 & 1.07 \\
\hline PEGS $^{2}$ & 20 & 1.11 & 1.09 & 1.08 \\
\hline
\end{tabular}




\begin{tabular}{|c|c|c|c|c|}
\hline $\mathrm{PC}^{11}$ & 38 & 1.20 & 1.19 & 1.15 \\
\hline $\mathrm{PBzA}^{12}$ & 5 & 1.28 & 1.26 & 1.21 \\
\hline
\end{tabular}

${ }^{a}$ Content of cross-linking units in the linear precursor polymer. ${ }^{b}$ Weight-average molecular weight to number-average molecular weight ratio of the linear precursor as determined by SEC with traditional calibration. ${ }^{c}$ Weight-average molecular weight to number-average molecular weight ratio of the resulting intramolecular cross-linked nanoparticles as determined by SEC with traditional calibration. ${ }^{\mathrm{d}}$ Calculated from equation 10 by assuming $\beta \approx 0.88$.

\section{Concluding remarks}

By considering the change accompanying the irreversible, intramolecular collapse of individual polymer chains to fractal, soft nano-objects and its effect on hydrodynamic behavior a unifying picture emerges allowing to quantify the reduction observed by SEC with traditional calibration in both (apparent) molecular weight and polydispersity.

First, most of the available experimental data in the literature for intramolecular cross-linked nanoparticles covering different polymer chemistries follow a $M_{a p p} \propto M^{\beta}$ scaling-law where $\beta=\left(1+\alpha_{F}\right) /\left(1+\alpha_{L}\right), \alpha_{F}$ being a parameter related to the fractal and soft nature of the nanoparticle and $\alpha_{L}$ the Mark-Houwink-Sakurada exponent of the precursor polymer, respectively. Experimental values of $\beta$ are typically around $0.85-$ 0.88, as illustrated in Figures 1 and 2 for PS and PMMA nanoparticles, suggesting values of the fractal parameter $\alpha_{F}$ around $0.47-0.52$ and hence pointing to a hydrodynamic behavior of the nanoparticles "equivalent" to that of linear polymer chains in $\theta$-solvents. This behavior can be attributed to the partially collapsed nature of the soft nanoparticles since for totally compact, spherical particles a value of $\alpha_{F} \approx 0$ should be expected. Preliminary results from molecular dynamics simulations of the single-chain cross-linking process, that will be the subject of a separate paper, provide $\beta$ values in very good agreement with experimental data (e.g. $\beta=0.87$ for collapsed nanoparticles arising from precursor chains with a $20 \%$ of functional, cross-linking monomers). 
Second, the above $M_{a p p} \propto M^{\beta}$ power-law has a significant impact on the apparent polydispersity of the unimolecular nanoparticles. Hence, for a precursor displaying a log-normal $M W D$ function we find $P D I_{a p p}=(P D I)^{\beta^{2}}$ where $P D I$ and $P D I_{a p p}$ are the polydispersity index of the precursor and the unimolecular nanoparticles, respectively. Experimental data are consistent with this scaling-law although due to the inherent heterogeneous nature of the intramolecular cross-linking process and/or the potential presence of marginal cross-coupling products significant data scatter is observed for some systems.

Finally, we hope this work will contribute to stimulate an in-deep characterization by means of experiment and computer simulations of the complex intramolecular cross-linking process and, at the end, of the physics beyond unimolecular polymeric nanoparticles.

Acknowledgements. I. P.-B. thanks CSIC for her JAE-PREDOC grant. L. B. acknowledges financial support by Basque Excellence Research Center (BERC)Materials Physics Center. Authors gratefully acknowledge financial support from the Spanish Ministry of Science and Innovation, project MAT-22007-63681, the Basque Government, project IT-436-07 and Diputación de Gipuzkoa, project 2011-CIEN000085-01.

\section{References and notes}

(1) (a) Mackay, M. E.; Dao, T. T.; Tuteja, A.; Ho, D. L.; Horn, B. v.; Kim, H.-C.; Hawker, C. J. Nature Mater. 2003, 2, 762-766. (b) Ryu, J.-H., Jiwpanich, S., Chacko, R., Bickerton, S., and Thayumanavan, S. J. Am. Chem. Soc. 2010, 132, 8246-8247. (c) Oishi, M.; Nagasaki, Y. Nanomedicine-UK 2010, 5, 451-468. (d) Oria, L.; Aguado, R; Pomposo, J. A.; Colmenero, J. Adv. Mater. 2010, 22, 30383041 .

(2) Harth, E.; Horn, B. V.; Lee, V. Y.; Germack, D. S.; Gonzales, C. P.; Miller, R. D.; Hawker, C. J. J. Am. Chem. Soc. 2002, 124, 8653-8660.

(3) (a) Kopesky, E. T.; Haddad, T. S.; Cohen, R. E.; McKinley, G. H. Macromolecules 2004, 37, 8992-9004. (b) Tuteja, A.; Duxbury, P. M.; Mackay, 
M. E. Macromolecules 2007, 40, 9427-9434. (c) Tuteja, A.; Mackay, M. E.; Hawker, C. J., Horn, B. v. Macromolecules 2005, 38, 8000-8011. (d) Schmidt, R. G.; Gordon, G. V.; Dreiss, C. A.; Cosgrove, T.; Krukonis, V. J.; Williams, K.; Wetmore, P. M. Macromolecules 2010, 43, 10143-10151.

(4) (a) Wulff, G.; Chong, B.-O.; Kolb, U. Angew. Chem. Int. Ed. 2006, 45, 2955. (b) Liu, J.; Wulff, G. J. Am. Chem. Soc. 2008, 130, 8044-8054. (c) Takahashi, H.; Sawada, S.; Akiyoshi, K. ACS Nano, 2010, 5, 337-345.

(5) (a) Kim, H. J.; Lee, J.; Kim, T. H.; Lee, T. S.; Kim, J. Adv. Mater. 2008, 20, 1117-1121. (b) Adkins, C. T.; Muchalski, H.; Harth, E. Macromolecules 2009, 42, 5786-5792. (c) Perez-Baena, I.; Loinaz, I.; Padro, D.; Garcia, I.; Grande, H. J.; Odriozola, I. J. Mater. Chem. 2010, 33, 6916-6922.

(6) (a) Hamilton, S. K.; Harth, E. ACS Nano 2009, 3, 402-410. (b) Tamura, A.; Nagasaki, Y. Nanomedicine-UK 2010, 5, 1089-1102.

(7) Mecerreyes, D; Lee, V.; Hawker, C. J.; Hedrick, J. L.; Wursch, A.; Volksen, W.; Magbitang, T.; Huang, E.; Miller, R. D. Adv. Mater. 2001, 13, 204-208.

(8) Park, K. S.; Kim, D. Y.; Choi, S. K.; Suh, D. H. Jpn. J. Appl. Phys. 2003, 42, 3877-3880.

(9) Jiang, J; Thayumanavan, S. Macromolecules 2005, 38, 5886-5891.

(10) Taranekar, P.; Park, J. Y.; Patton, D.; Fulghum, T.; Ramon, G. J.; Asvincula, R. Adv. Mater. 2006, 18, 2461-2465.

(11) Cherian, A. E.; Sun, F. C.; Sheiko, S. S.; Coates, G. W. J. Am. Chem. Soc. 2007, $129,11350-11351$.

(12) Croce, T. A.; Hamilton, S. K.; Chen, M. L.; Muchalski, H.; Harth, E. Macromolecules 2007, 40, 6028-6031.

(13) Ruiz de Luzuriaga, A.; Ormategui, N.; Grande, H. J.; Odriozola, I.; Pomposo, J. A.; Loinaz, I. Macromol. Rapid Commun. 2008, 29, 1156-1160.

(14) Njikang, G.; Liu, G.; Curda, S. A. Macromolecules 2008, 41, 5697-5702.

(15) Radu, J. E. F.; Novak, L.; Hartmann, J. F.; Beheshti, N.; Kjoniksen, A.-L.; Nystrom, B.; Borbely, J. Colloid Polym. Sci. 2008, 286, 365-376.

(16) Cheng, L.; Hou, G.; Miao, J.; Chen, D.; Jiang, M.; Zhu, L. Macromolecules 2008, $41,8159-8166$.

(17) Beck, J. B.; Killops, K. L.; Kang, T.; Sivanandan, K.; Bayles, A.; Mackay, M. E.; Wooley, K.; Hawker, C. J. Macromolecules 2009, 42, 5629-5635.

(18) Adkins, C. T.; Muchalski, H.; Harth, E. Macromolecules 2009, 42, 5786-5792. 
(19) Foster, E. J.; Berda, E. B.; Meijer, E. W. J. Am. Chem. Soc. 2009, 131, 69646966.

(20) Berda, E. B.; Foster, E. J.; Meijer, E. W. Macromolecules 2010, 43, 1430-1437.

(21) Bodnar, M.; Hartmann, J.; Borbely, J. Biomacromolecules 2006, 7, 3030-3036.

(22) Seo, M.; Beck, B. J.; Pulusse, J. M.; Hawker, C. J.; Kim, S. Y. Macromolecules 2008, 41, 6413-6418.

(23) Van-der-Ende, A. E.; Kravitz, E.; Harth, E. J. Am. Chem. Soc. 2008, 130, 87068713.

(24) Tao, J.; Liu, G. Macromolecules 1997, 30, 2408-2411.

(25) Since intermolecular reactions give rise indefectibly to an increase in the molecular weight, a molecular weight reduction is a clear signature of intramolecular chain collapse (in absence of secondary reactions during crosslinking like depolymerization, chain scission, etc.). Even the presence of two-, three-, four- and even five-chain cross-linked species can be detected by SEC, see ref. 16 for illustration.

(26) Size Exclusion Chromatography. Mori, S; Barth, H. G. Springer, 1999.

(27) (a) Methods in Molecular Biophysics: Structure, Dynamics, Function. Serdyuk, I. N.; Zaccai, N. R.; Zaccai, J. Cambridge Univ. Press, 2007. (b) Physical Properties of Polymers Handbook. Mark, J. E. Springer, 2007.

(28) Fetters, L. J.; Hadjichristidis, N.; Lindner, J. S.; Mays, J. W. J. Phys. Chem. Ref. Data 1994, 23, 619-640.

(29) (a) Principles of Polymer Chemistry. Flory, P. J. Cornell Univ. Press, 1953. (b) Polymer Handbook, $3^{\text {rd }}$ Edition, Brandrup, J; Immergut, E. H. Wiley, 1989.

(30) (a) Hild, G.; Strazielle, C.; Rempp, P. Eur. Polym. J. 1983, 19, 721-727. (b) Jang, S. S.; Cagin, T.; Goddard, W. A. J. Chem. Phys. 2003, 119, 1843-1854.

(31) Chen, Y. J.; Li, J.; Hadjichristidis, N.; Mays, J. M. Polym. Bull. 1993, 30, 575578.

(32) Glassner, M.; Blinco, J. P.; Barner-Kowollik, C. Macromol. Rapid Commun. 2011, 32, 724-728.

(33) The Log-normal Distribution. Aitchison, J.; Brown, J. A. C. Cambridge University Press, 1957.

(34) Liu, J. W.; Mackay, M. E.; Duxbury, P. M. Macromolecules 2009, 42, 8534-8542. 
FOR “TABLE OF CONTENTS” USE ONLY

On the Apparent SEC Molecular Weight and Polydispersity Reduction upon Intramolecular Collapse of Polydisperse Chains to Unimolecular Nanoparticles

José A. Pomposo, Irma Perez-Baena, Lorea Buruaga, Angel Alegría, Angel J. Moreno and Juan Colmenero

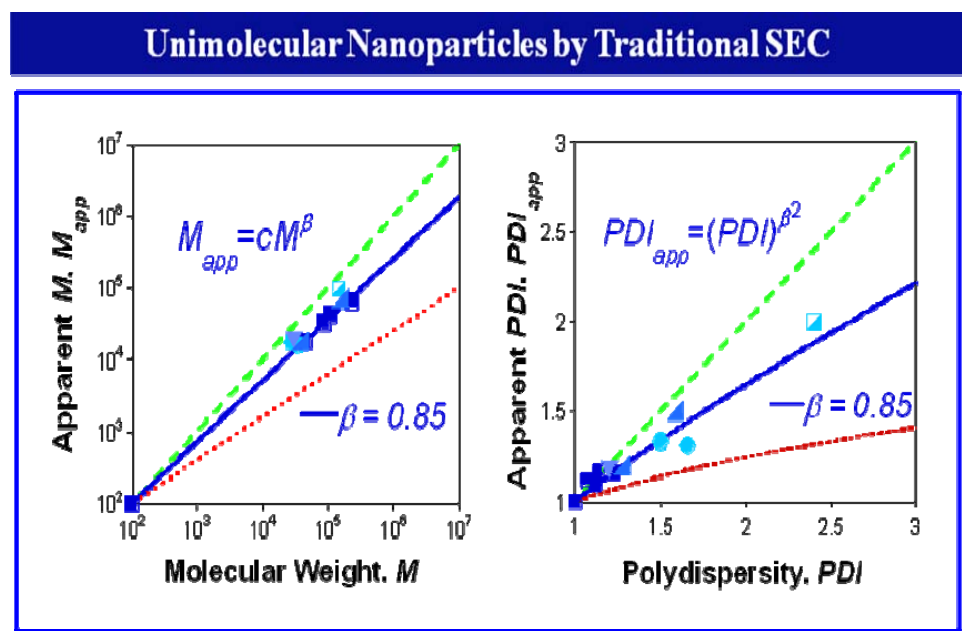

